\title{
Online Impedance Estimation in AC Grids Considering Parallel-Connected Converters
}

\author{
Andrés Suárez, Cristian Blanco, Pablo García, Ángel Navarro-Rodríguez, Jose Manuel Cano Rodríguez \\ Dept. of Electrical, Electronic, Computers and Systems Engineering \\ University of Oviedo Gijón, 33204, Spain \\ e-mail: suarezandres@uniovi.es, blancocristian@uniovi.es, garciafpablo@uniovi.es, navarroangel@uniovi.es, jmcano@uniovi.es
}

\begin{abstract}
This paper addresses the estimation of the grid impedance under unbalanced conditions in AC Grids with power converter operating in parallel. A Pulsed Signal Injection (PSI) approach is employed for online estimating the grid impedance using an Recursive Least Square (RLS) algorithm. The method proposes here extends previous works focused on the impedance measurement and identification for pure passive grids. The most important contribution of the proposal can thus be summarized as the presentation of an online impedance technique suitable to be used in distribution grids having other power converters connected near the Point of Common Coupling (PCC). The proposal is valid both for balanced and unbalanced conditions.
\end{abstract}

\section{INTRODUCTION}

Interfacing Voltage Source Converters (VSC) with the AC grid requires a very precise design of the current controller in order to fulfill the currents requirements of the grid. For this reason it is critical to have a deep knowledge of the dynamic model between the converter output voltage and the resulting grid current. The dynamics of this model are affected by the filter topology, i.e. L, LC, LCL, and the disturbance decoupling capability depending on the number and the position of the voltage and current sensors [1], [2].

There are multitude methods for grid impedance estimation, an initial classification may be according the ones that require additional dedicated hardware to perform the estimation and the ones that not. In first group [3], [4] use signal injection and frequency based techniques to estimate the impedance over a wide frequency range. Regarding the second group, impedance estimation could be implemented using two different approaches:

- Model-based techniques, where the transfer function between the voltage and the current is used for the estimation. Examples of these techniques are present in the literature, i.e. the use of the LCL filter resonance proposed in [5] for grid estimation. However the presence of two resonant frequencies when reactive power passive

The present work has been partially supported by the predoctoral grants program Severo Ochoa for the formation in research and university teaching of Principado de Asturias PCTI-FICYT under the grant ID BP14-135. This work also was supported in part by the Research, Technological Development and Innovation Program Oriented to the Society Challenges of the Spanish Ministry of Economy and Competitiveness under grant ENE2016-77919-R, ENE2014-52272-R, by the European Union through ERFD Structural Funds (FEDER) and by the government of Principality of Asturias, under IDEPA grant 2017 Thyssen SV-PA-17-RIS3-3. is added at the PCC is the main limitation of this strategy. In [6] a experimentally supported method is proposed for estimating the equivalent inductance and resistance of the grid. It is based on the closed-loop transient response, nonetheless this method does not include the operation under unbalanced grid conditions and does not show the response to sudden changes on the grid impedance, which is critical for islanding detection. The use of Kalman filters for grid estimation are other solutions within this approach. In [7] grid impedance is estimated based on the grid harmonics and the impedance at different frequencies, this study is only supported with simulations. In [8] a similar approach is presented; the estimated coefficients are online updated using an adaptive linear neuron (ADALINE) algorithm, this model requires to measure grid current and voltages but it is experimentally demonstrated under balanced conditions. In [9], grid inductance is estimated using two consecutive samples of the grid current within a switching period. The estimation method is based on the discrete-time model at the grid frequency and, as recognized by the authors, the method is only valid for the inductive component. Moreover, the operation is only demonstrated for two different inductance values and unbalanced conditions are not considered.

- Signal-injection based techniques use an additional excitation for tracking the system response [10]-[17]. For the excitation signal, several approaches have been proposed:

1) Pulsed Signal Injection (PSI) [3], [17]-[19]

2) High Frequency Signal Injection (HFSI) at constant high frequency [12], [13], [20]-[22];

3) Current regulator reaction [13];

4) Low Frequency Signal Injection (LFSI) [14], [23], [24];

5) Binary Sequence Signal Injection (BSSI) [15], [25], [26].

For the PSI methods studied in literature, the results presented in [18] are closed to the ones presented in the present work, but deeper focused on a pure inductive and a balanced three phase impedance. Authors of [17] proposed a solution able to estimate grid admitance considering also power con- 
verters connected in parallel, however the activation of the pulse injection is not fully described and results were only tested under real-time emulation by an OPAL-RT simulator. Other approaches as [19] proposed a method with a larger computational burden and memory requirements than the one in the present work. Results in [3] are obtained under the absence of fundamental excitation. HFSI methods present some issues regarding the selection of the frequency range, since it is possible to produce a reaction in any other active power filter (APF) connected to the same PCC, compensating the high frequency and depreciating the estimation. Organizing LFSI methods depending into those adding an additional excitation signal at a given frequency and analyzing the response of the grid at that frequency to obtain the impedance, it is highlighted the results obtained in [11], [23], where it is used a $75 \mathrm{~Hz}$ current signal. The continuous injection of the signal may produced distortion in the components and the impedance is only estimated for the frequency of the signal injected. It has also to be considered that this low-frequency signal is suitable to be compensated by the own dynamic response of the current regulators, reacting at that frequency and compensating the disturbance signal, which reduces the effectiveness of the method. On the other hand, considering the LFSI methods that modifies the commands delivered by the converter, a solution modifying the $P$ and $Q$ command to analyze the response of the system and to estimate the impedance is proposed in [24], however the results are not validated under real situation neither unbalanced conditions and present a drawback considering the coupling terms between the regular operational condition and the ones induced to perform the estimation. Other approaches for estimation based on frequency methods, such BSSI, which is based on injecting a pulse-train of signals and analyzing the response are also present in literature. This approach requires larger processing time and computational burden than PSI methods and they are not tested under unbalanced grid conditions.

This paper presents a strategy for grid estimation, based on the PSI technique, considering a distribution grid with parallel connected converters and operated under balanced and unbalanced conditions. The proposed PSI method injects a pulse at the zero crossing of each three phase voltages, which reduce the THD of the system when compared to other strategies, and the estimation is based on the performance of a Recursive Least Square (RLS) algorithm. The proposed strategy is supported with experimental results. The purpose of the study is to analyze the behavior of the grid impedance under these conditions and evaluate how is affected by the operation of additional converters connected to the same grid. The study also addresses the behavior of the estimator under those conditions. The contributions of this work can be summarized as: 1) online identification of the grid impedance, including unbalance conditions; 2) Definition of a model to evaluate the grid impedance under balanced and unbalanced condition; 3) identification and evaluation of the grid impedance estimation with multiple converters connected to the same grid.

This paper is organized as follows. In Section II is presented the impedance modeling technique, which highlight a coupling between the impedance of the system and the sequences generated under an unbalance in the system. Simulation and also experimental results related to the impedance model are included in this part. In Section III the PSI strategy is presented. Section IV describes the algorithm of the RLS used for the estimation, as well as the simulation results. Section $\mathrm{V}$ presents the laboratory results obtained and finally Section VI summarizes the conclusions of the work.

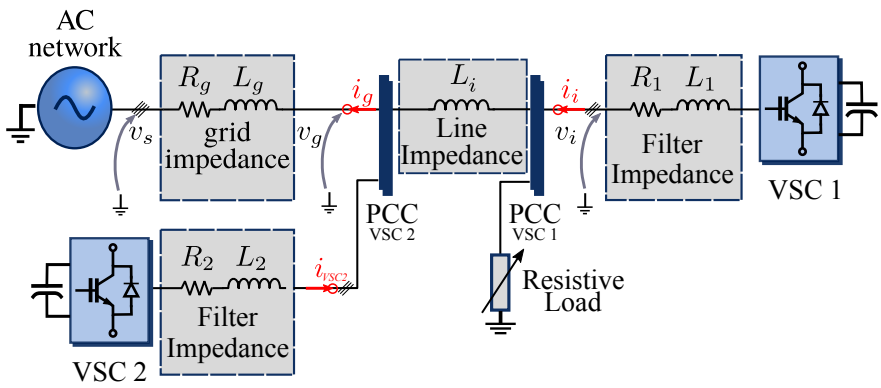

Fig. 1. Schematic of the considered system. Two power converters and a resistive load are considered for the present work. VSC1 represents the converter implementing the PSI method.

\section{System Modeling ANd IMPEDANCE DeFINITION}

The equations for the system presented in FIg.1 are suitable to be defined both in stationary $(\alpha, \beta, 0)$ or synchronous $(d, q, 0)$ reference frame. For this work, $\alpha, \beta, 0$ reference was selected, following same procedure as stated in [27], since it leads to a simpler formulation in the presence of unbalances. The objective of this work is to define a model suitable for the identification of the grid impedance. This impedance contribution may be split into two parts; a first one imposed by the cabling and the connection/disconnection of passive loads and a second active part that is determined by the operational mode of the power converters, including harmonic generation or compensation.

\section{A. Equivalent Impedance of Passive Loads}

Voltage equation at the PCC, considering a RL filter for interconnection between the grid and the converter, is defined as

$$
\mathbf{v}_{\alpha \beta}=\mathbf{R}_{\alpha \beta} \mathbf{i}_{\alpha \beta}+\mathbf{L}_{\alpha \beta} \frac{d \mathbf{i}_{\alpha \beta}}{d t}
$$

By considering the angle of the impedance as the angle for the spatial orientation in the stationary reference frame, the equivalent expression for the statical impedance as seen by the converter may be defined as (2).

$$
\mathbf{Z}_{\alpha \beta_{i}}=\mathbf{R}_{\alpha \beta_{i}}+\mathbf{j} \omega_{e} \mathbf{L}_{\alpha \beta_{i}}=\left(\begin{array}{cc}
Z_{\alpha \alpha i} & Z_{\alpha \beta_{i}} \\
Z_{\alpha \beta_{i}} & Z_{\beta \beta_{i}}
\end{array}\right)
$$

Subscript $i$ is related to each individual impedance seen from the PCC. In case the impedance is balanced, diagonal terms $\left(Z_{\alpha \alpha i}\right.$ and $\left.Z_{\beta \beta_{i}}\right)$ are equal and cross-coupling between phases, represented by non diagonal terms $\left(Z_{\alpha \beta_{i}}\right)$, are not present. For the case of unbalance, expression (2) can be 
rotated to a common $\alpha \beta$ reference frame, with $n$ impedance elements, leading to (3).

$$
\begin{aligned}
\mathbf{Z}_{\alpha \beta 0} & =\sum_{i=1}^{n}\left[\Sigma Z_{i}\left(\begin{array}{ll}
1 & 0 \\
0 & 1
\end{array}\right)+\Delta Z_{i}\left(\begin{array}{cc}
\cos \theta_{e}^{i} & -\sin \theta_{e}^{i} \\
-\sin \theta_{e}^{i} & -\cos \theta_{e}^{i}
\end{array}\right)\right. \\
& \left.+Z_{\alpha \beta_{i}}\left(\begin{array}{cc}
-\sin \theta_{e}^{i} & \cos \theta_{e}^{i} \\
\cos \theta_{e}^{i} & \sin \theta_{e}^{i}
\end{array}\right)\right]
\end{aligned}
$$

where $\Sigma Z_{i}=\frac{Z_{\alpha \alpha_{i}}+Z_{\beta \beta_{i}}}{2}, \Delta Z_{i}=\frac{Z_{\alpha \alpha_{i}}-Z_{\beta \beta_{i}}}{2}$, and $\theta_{e}^{i}$ is the spatial angular phase of the unbalance impedance. For a balanced system, only the matrix terms depending on $\Sigma Z_{i}$ will remain. Relationship with phase impedances are: $\Sigma Z_{i}=$ $\frac{z a_{i}+z b_{i}+z c_{i}}{3}, \Delta Z_{i}=\frac{z a_{i}+a \cdot z b_{i}+a^{2} \cdot z c_{i}}{3}$, where $a=e^{j 2 \pi / 3}$.

Figure 2 shows the results of the variation in the equivalent impedance of the system according to variation induced in the load. Initially, for a voltage level of $400 \mathrm{~V}$, the equivalent impedance of the system, $\Sigma Z$, is $18 \Omega$, for that case, the system is balanced and consequently, only positive sequence current is present on the system, with peak value of $22 \mathrm{~A}$, the rest of the components are zero. At instant $t=0.125 \mathrm{~s}$, there is a change in one of the impedance phases, increasing a $50 \%$ its nominal value. As consequence of this unbalance in the impedance, negative and homopolar current components are flowing through the system. The value for the components of the impedance are $\Sigma Z=21.48 \Omega$ and $\Delta Z=2.791 \Omega$, the angle of the unbalance is reflected through $\theta$ with a value of $-8.5^{\circ}$. In the next step of the simulation at $t=0.25 \mathrm{~s}$, the impedance of the three phases are increased in $50 \%$ its value. This change is produced at same time and magnitude in the three phases, so that there is not unbalance, either negative sequence components. For same reason, $\Delta Z$ component is zero and the impedance variation is only affecting the $\Sigma Z$ component. In the last part of the simulation a change in two phases is made simultaneously producing an equivalente impedance of $\Delta Z=5.2 \Omega$ and a $\Sigma Z=27 \Omega$ with an angle for the unbalance of $150^{\circ}$.

By considering the overall grid impedance dominated by the resistance and inductance terms, it can be expressed as

$$
\begin{aligned}
\Sigma Z_{i} & =\Sigma R_{i}+j \omega_{e} \Sigma L_{i} \\
\Delta Z_{i} & =\Delta R_{i}+j \omega_{e} \Delta L_{i} \\
\mathbf{Z}_{\alpha \beta i} & =\mathbf{R}_{\alpha \beta i}+j \omega_{e} \mathbf{L}_{\alpha \beta i}
\end{aligned}
$$

where, $\Sigma R_{i}=\frac{R_{\alpha \alpha_{i}}+R_{\beta \beta_{i}}}{2}$, and $\Sigma L_{i}=\frac{L_{\alpha \alpha_{i}}+L_{\beta \beta_{i}}}{2}, \Delta R_{i}=$ $\frac{R_{\alpha \alpha_{i}}-R_{\beta \beta_{i}}}{2}, \Delta L_{i}=\frac{L_{\alpha \alpha_{i}}-L_{\beta \beta_{i}}}{2}$. From here it can be obtained a voltage expression (5),

$$
\mathbf{v}_{z e q}{ }^{\alpha \beta}=\mathbf{v}_{g}{ }^{\alpha \beta}-\mathbf{v}_{s}{ }^{\alpha \beta}=\mathbf{R}_{\alpha \beta} \mathbf{i}_{g}{ }^{\alpha \beta}+\mathbf{L}_{\alpha \beta} \frac{d \mathbf{i}_{g}^{\alpha \beta}}{d t}
$$

where $\mathbf{v}_{z e q}{ }^{\alpha \beta}$ is the voltage drop vector across the overall equivalent impedance, $\mathbf{v}_{g}{ }^{\alpha \beta}$ and $\mathbf{v}_{s}{ }^{\alpha \beta}$ are the PCC voltage and the grid voltage vectors (see Fig. 1), and $\mathbf{i}_{g}{ }^{\alpha \beta}$ is the grid current vector. $\mathbf{L}_{\alpha \beta}$ and $\mathbf{R}_{\alpha \beta}$ are, respectively, the sum of

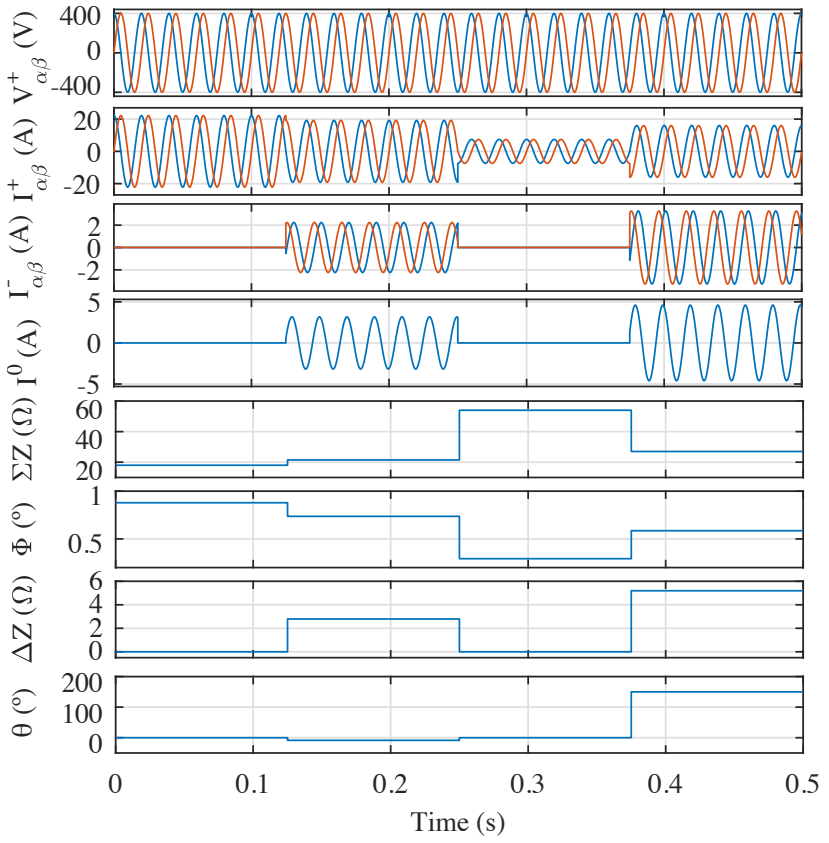

Fig. 2. From top to bottom, 1) Positive Sequence of AC-Grid Voltage in the $\alpha \beta$ reference frame; 2) Positive Sequence of Current in the $\alpha \beta$ reference frame; 3) Negative Sequence of Current in the $\alpha \beta$ reference frame; 4) Homopolar Current; 5) Equivalent impedance of $\Sigma Z$ according to expression (3); 6) Angle of $\Sigma Z$; 7) $\Delta Z$, Equivalent impedance of the system under an unbalance situation; 8) $\theta$, Angle of the unbalance

the inductance and resistance matrices for the different grid impedances as expressed in (4c).

\section{B. Equivalent Impedance of Active Loads}

Impedance identification in those applications considering power converters, Fig.1, requires considering the effect of the converter operating point in the equivalent impedance. Power converters, working as STATCOMs, behave as an active filter, modifying their equivalent impedance to achieve the reactive power requirements of the system. Consequently, it is need to estimate an initial approach of the converter impedance working under such operation. In Fig.1, VSC2 was connected to the grid in STATCOM mode. The $d$ component of the control loop is dedicated to control the dc-link of the converter and $q$ component is devoted to inject different levels of reactive current into the grid. Measuring voltage and current at the converter PCC and implementing the modeling technique previously described, it is possible to estimate an initial value for the impedance of the converter. Figure 3 represents the real impedance of VSC2 for a range of frequencies and current injection from 6 to $11 \mathrm{~A}$. According the results, the whole impedance value decreases proportionally to the current and the frequency rise. The results of the same experiment are presented in Fig. 4 in time domain and for the main frequency of the grid. In this case, the inductance part of the impedance is kept constant for same current level. Values of the impedance are $50 \mathrm{mH}$ for the highest current of $11 \mathrm{~A}$ and $70 \mathrm{mH}$ for the lowest current at $6 A$. The resistive part varies much less 

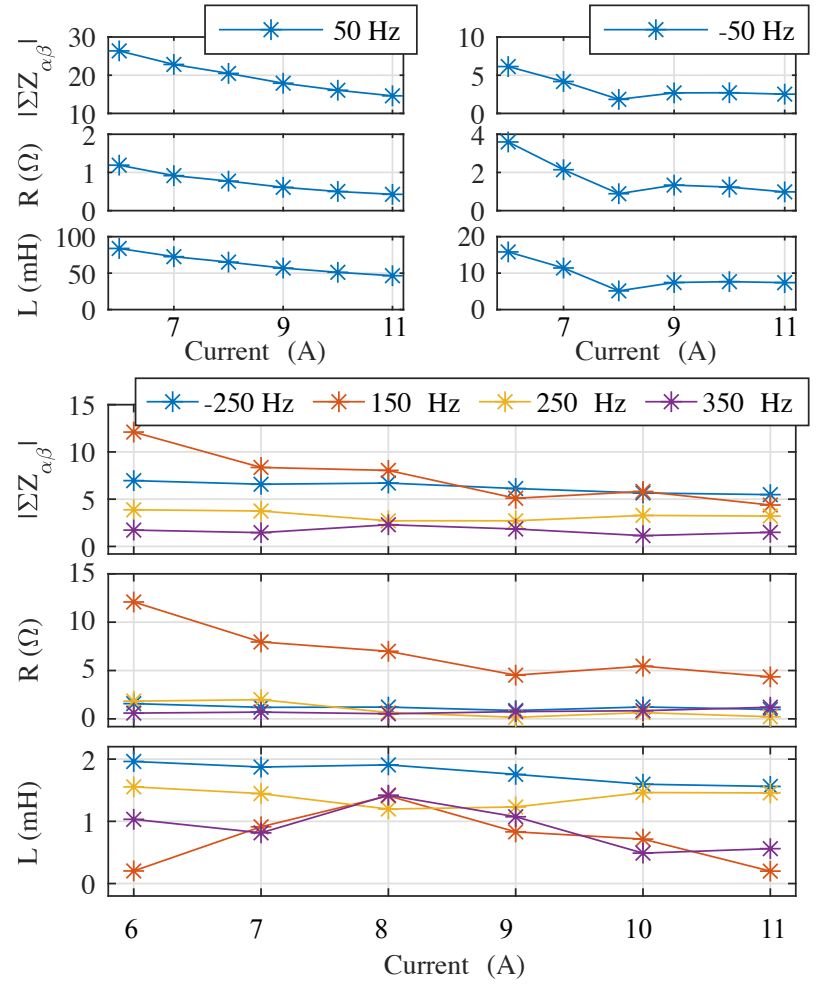

Fig. 3. From top to bottom: 1)In the left; a) Equivalent impedance of the system at different reactive current values for a frequency of $50 \mathrm{~Hz} ; \mathrm{b}$ ) Equivalent resistance of the system for different values of reactive current at $50 \mathrm{~Hz} ; \mathrm{c}$ ) Inductance value of the system at a frequency of $50 \mathrm{~Hz}$ for different reactive current injection. 2) In the right: Idem 1) at $-50 \mathrm{~Hz}$. 3) In the bottom: a) Equivalent impedance of the system at different frequencies and current injection; b) Equivalent resistance of the system at different frequencies and current injection; c) Inductance equivalent for different frequencies and current injection

according the currents, keeping constant at value of $1 \Omega$ for the whole set of currents.

Since those currents are balanced, there is no presence of negative or homopolar sequence components, so the results are feasible to identify the $\Sigma Z$ component from expression (3). The rest of non-diagonal components are result of interaction between non-positive sequence components in the system. Theoretical relationship between sequences are illustrated in Fig.5. As said above, under balanced situation, only $\Sigma Z$ is interacting at main frequency. Only under an unbalance situation, $\Delta Z$ is contributing to the cross-term element.

According [27] and expanding (1) for every single impedance in the system, it is possible to obtain a relation between positive and negative sequence components and the system impedance, as it states in (6)

$$
\begin{aligned}
\mathbf{i}_{\alpha \beta} & =\left[\mathbf{Z}_{\alpha \beta}\right]^{-1} \cdot \mathbf{v}_{\alpha \beta} \\
& =\mathbf{v}_{\alpha \beta} \cdot \frac{1}{\Sigma Z_{i}^{2}-\Delta Z_{i}^{2}} \\
& \cdot\left[\left(\begin{array}{cc}
\Sigma Z_{i} & 0 \\
0 & \Sigma Z_{i}
\end{array}\right)+\Delta Z_{i}\left(\begin{array}{cc}
-\cos \theta_{e}^{i} & \sin \theta_{e}^{i} \\
\sin \theta_{e}^{i} & \cos \theta_{e}^{i}
\end{array}\right)\right] \\
& =\frac{\Sigma Z_{i}}{\Sigma Z_{i}^{2}-\Delta Z_{i}^{2}} \cdot V_{\alpha \beta} e^{j \omega t}-\frac{\Delta Z_{i}}{\Sigma Z_{i}^{2}-\Delta Z_{i}^{2}} \cdot V_{\alpha \beta} e^{-(j \omega t+\theta)}
\end{aligned}
$$

$$
\begin{gathered}
{\left[\begin{array}{c}
i_{\alpha}^{(+)} \\
i_{\beta}^{(+)} \\
i_{\alpha}^{(-)} \\
i_{\beta}^{(-)}
\end{array}\right]=} \\
\frac{1}{\Sigma Z_{i}^{2}-\Delta Z_{i}^{2}}\left[\begin{array}{cccc}
\Sigma Z_{i} & 0 & -\Delta Z_{i} & 0 \\
0 & \Sigma Z_{i} & 0 & -\Delta Z_{i} \\
-\Delta Z_{i} & 0 & \Sigma Z_{i} & 0 \\
0 & -\Delta Z_{i} & 0 & \Sigma Z_{i}
\end{array}\right]\left[\begin{array}{c}
v_{\alpha}^{(+)} \\
v_{\beta}^{(+)} \\
v_{\alpha}^{(-)} \\
v_{\beta}^{(-)}
\end{array}\right]
\end{gathered}
$$

Considering the power expression depicted in (8), where the constant value are terms associated to the positive sequence and the other terms the interacting between different sequences.

$$
\begin{aligned}
{\left[\begin{array}{l}
p \\
q
\end{array}\right] } & =\left[\begin{array}{l}
v_{\alpha}^{+} \cdot\left(i_{\alpha}^{+}+i_{\alpha}^{-}\right)+v_{\beta}^{+} \cdot\left(i_{\beta}^{+}+i_{\beta}^{-}\right) \\
v_{\beta}^{+} \cdot\left(i_{\alpha}^{+}+i_{\alpha}^{-}\right)-v_{\alpha}^{+} \cdot\left(i_{\beta}^{+}+i_{\beta}^{-}\right)
\end{array}\right] \\
& =\left[\begin{array}{c}
\bar{P}+\widetilde{P} \\
\bar{Q}+\widetilde{Q}
\end{array}\right]
\end{aligned}
$$


Combination of (7) and (8) allows to establish a relationship between every term of the equivalent impedance and power. Multiple combinations are possible, however for this work it was only considered the case of negative sequence current, resulting in (9)

$$
\left[\begin{array}{c}
\bar{P} \\
\widetilde{P} \\
\bar{Q} \\
\widetilde{Q}
\end{array}\right]=\frac{1}{\Sigma Z_{i}^{2}-\Delta Z_{i}^{2}} \cdot\left[\begin{array}{c}
\Sigma Z_{\alpha}+\Sigma Z_{\beta} \\
-\Delta Z_{\alpha}-\Delta Z_{\beta} \\
-\Sigma Z_{\alpha}+\Sigma Z_{\beta} \\
\Delta Z_{\alpha}-\Delta Z_{\beta}
\end{array}\right] \cdot\left[\begin{array}{c}
v_{\alpha}^{+2} \\
v_{\beta}^{+2}
\end{array}\right]
$$

The term that relates every term in the graph To estimate those components inside the system of Fig.1, the current control of the converter was forced to inject harmonic components at $-50 \mathrm{~Hz}$. In this way it is possible to see a negative sequence current flowing through the system. Figure 6 presents the results of the negative sequence current injection. Voltage at

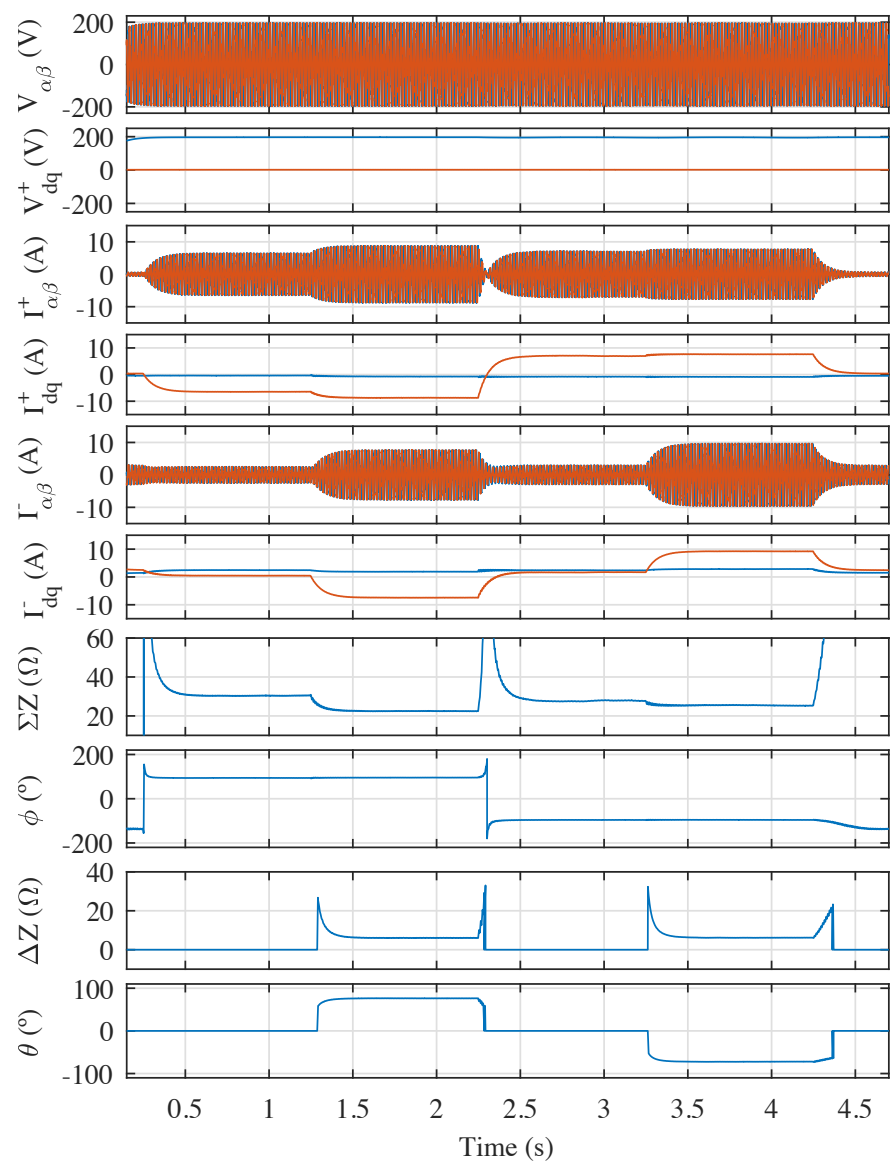

Fig. 6. From top to bottom: 1) Grid voltage in $\alpha \beta$ reference frame; 2) Grid voltage in $d q$ reference frame; 3 ) Positive sequence of converter current in $\alpha \beta$ reference frame; 4) Positive sequence of converter current in $d q$ reference frame; 5) Negative sequence of converter current in $\alpha \beta$ reference frame; 6) Negative sequence of converter current in $d q$ reference frame; 7) $\Sigma Z$ component of the equivalent converter impedance; 8) Angle of the $\Sigma Z$ component, expressed in degrees; 9) $\Delta Z$ component of the equivalent converter impedance; 10) $\theta$ angle of the unbalance, expressed in degrees.

the grid was set to $200 \mathrm{~V}$, at instant $0.125 \mathrm{~s}$ the converter started to inject $-7 A$ of positive sequence current into the system. That reaction is perceptible at the $\Sigma Z$ component of the impedance that is already set at value $30.23 \Omega$ with an angle of $90^{\circ}$, the rest of the components are kept to zero. At instant $1.25 \mathrm{~s}$, the negative component of the current is set to $-9 \mathrm{~A}$. At that instant the $\Sigma Z$ decreases to $22.45 \Omega$ and $\Delta Z$ increases to $25.46 \Omega$ and the angle the unbalance, $\theta$, is $76^{\circ}$. In the next step of the experiment, $t=2.25 \mathrm{~s}$, the positive sequence current starts to inject $7 A, \Sigma Z$ reaches the previous value of $30.23 \Omega$ but its angle $\phi$ changes to $-90^{\circ}$; consequently, since there is not any negative sequence component, $\Delta Z$ is zero. At instant $t=3.25 \mathrm{~s}$, the negative sequence current is set to $9 \mathrm{~A}$. The magnitude of $\Delta Z$ increases to the same previous value of $25.46 \Omega$ and same happened to angle that has changed the direction and now it is $-76^{\circ}$.

\section{Pulsed Signal InJection}

According the injection parameters, there are multitude of PSI strategies which can be implemented. The one selected in this paper is implemented in the $d q$ reference frame. The signal is injected aligned with the zero crossing of the grid voltage, detected by the PLL, in order to minimize the voltage distortion, Fig. 7 shows a detailed view about the injection mechanism.

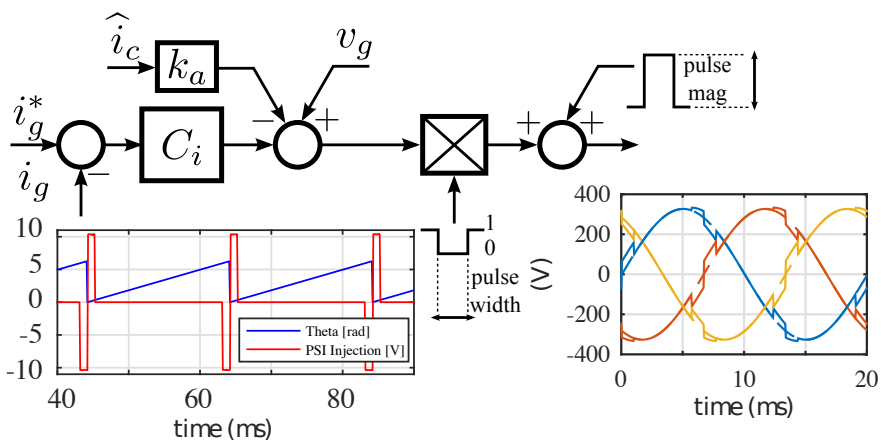

Fig. 7. PSI Loop implementation. The pulse injection is synchronized with respect to the grid voltage zero crossing and it is added in the $q$ component.

The pulses are injected by modifying the duty cycle provided by the current controller. The pulse is added to the $q$ component of the fundamental voltage. The magnitude and duration of the pulse may contribute to ease the detection of the impedance but also might increase the current THD, for this reason it is convenient to establish a trade-off between duration and also magnitude. The values presented in Table I have been used. Fig. 7 illustrates the strategy chosen for the experimental analysis. It is important to highlight the fact that since the pulse injection is simmetrical with respect to the zero crossing , thus resulting in a zero average voltage error. During the experimental analysis the injection was implemented in the $q$ component of the voltage, since the $d$ component is responsible to command the charge of the DC-Link.

\section{RLS ALGORITHM IMPLEMENTATION}

In this paper, the estimation of the system parameters is carried out by using an RLS approach [18], [28]. Starting from 
equation (5) and according [29], the discrete approximation of the grid current in $\alpha \beta$ reference frame, employing Tustin method and a sample time $T_{s}$ is

$$
\begin{aligned}
i_{g_{[k]}}^{\alpha} & =a_{1}^{\alpha} \cdot i_{g_{[k-1]}^{\alpha}}^{\alpha}+a_{2}^{\alpha} \cdot i_{g_{[k]}}^{\beta}+a_{3}^{\alpha} \cdot i_{g_{[k-1]}^{\beta}}^{\beta} \\
& +b_{0}^{\alpha}\left(v_{\alpha[k]}+v_{\alpha[k-1]}\right) \\
i_{g_{[k]}}^{\beta} & =a_{1}^{\beta} \cdot i_{g_{[k-1]}^{\beta}}^{\beta}+a_{2}^{\beta} \cdot i_{g_{[k]}^{\alpha}}^{\alpha}+a_{3}^{\beta} \cdot i_{g_{[k-1]}^{\alpha}}^{\alpha} \\
& +b_{0}^{\beta}\left(v_{\beta[k]}+v_{\beta_{[k-1]}}\right)
\end{aligned}
$$

where

$$
\begin{array}{rrr}
a_{1}^{\alpha}=\frac{\frac{2}{T_{s}} L_{\alpha \alpha}-R_{\alpha \alpha}}{\frac{2}{T_{s}} L_{\alpha \alpha}+R_{\alpha \alpha}}, & a_{2}^{\alpha}=-\frac{\frac{2}{T_{s}} L_{\alpha \beta}+R_{\alpha \beta}}{\frac{2}{T_{s}} L_{\alpha \alpha}+R_{\alpha \alpha}} \\
a_{3}^{\alpha}=\frac{\frac{2}{T_{s}} L_{\alpha \beta}+R_{\alpha \beta}}{\frac{2}{T_{s}} L_{\alpha \alpha}+R_{\alpha \alpha}}, & b_{0}^{\alpha}=\frac{1}{\frac{2}{T_{s}} L_{\alpha \alpha}+R_{\alpha \alpha}} \\
a_{1}^{\beta}=\frac{\frac{2}{T_{s}} L_{\beta \beta}-R_{\beta \beta}}{\frac{2}{T_{s}} L_{\beta \beta}+R_{\beta \beta}}, & a_{2}^{\beta}=-\frac{\frac{2}{T_{s}} L_{\alpha \beta}+R_{\alpha \beta}}{\frac{2}{T_{s}} L_{\beta \beta}+R_{\beta \beta}} \\
a_{3}^{\beta}=\frac{\frac{2}{T_{s}} L_{\alpha \beta}-R_{\alpha \beta}}{\frac{2}{T_{s}} L_{\beta \beta}+R_{\beta \beta}}, & b_{0}^{\beta}=\frac{1}{\frac{2}{T_{s}} L_{\beta \beta}+R_{\beta \beta}}
\end{array}
$$

and $v_{\alpha}, v_{\beta}$ represent the components of the difference between the PCC and the grid voltages. From (10), (11) the values for the resistance and inductance terms can be obtained as (12).

$$
\begin{aligned}
R_{x x}=\frac{1-a_{1}^{x}}{2 b_{0}^{x}}, & L_{x x}=\frac{T_{s}}{4} \frac{1+a_{1}^{x}}{b_{0}^{x}}, \\
R_{x y}=-\frac{a_{2}^{x}+a_{3}^{x}}{2 b_{0}^{x}}, & L_{x y}=\frac{T_{s}}{4} \frac{a_{3}^{x}-a_{2}^{x}}{b_{0}^{x}}
\end{aligned}
$$

where $x, y$ could be either $\alpha$ or $\beta$.

The RLS algorithm will allow to estimate the resistances and inductances in (12) by determining the values of the coefficients $a_{i}^{x}$ and $b_{j}^{x}$. The difference between the measured converter current, $i_{i[k]}^{x}$, and the one calculated by the estimator, $\widehat{i}_{i[k]}^{x}$, leads to the error driving the RLS update. Since it is assumed that the grid voltage is stiff enough, it is not necessary to consider any effect due to the pulse injection, so it is possible to decoupling the unknown grid voltage $\mathbf{v}_{s}^{\alpha \beta}$, by only considering the current induced by the pulse injection.

The least squares problem is formulated as in [29], in recursive form using the equations (13)-(16). The system equations are represented by defining the variables and coefficients vectors, $\mathbf{X}_{[k]}^{x}, \mathbf{W}_{[k]}^{x}$, as (17) and (18) respectively, where superscript $x$ could be either $\alpha$ or $\beta$. The estimated RLS current, $\hat{i}_{g[k]}^{x}$, is determined by the product $\mathbf{W}_{[k-1]}^{x} \cdot \mathbf{X}_{[k]}^{x}$ in (13). All the variables names are referred to those shown in Fig. 1.

$$
\begin{aligned}
\alpha_{[k]}^{x} & =i_{g[k]}^{x}-\mathbf{W}_{[k-1]}^{x} \cdot \mathbf{X}_{[k]}^{x} \\
\mathbf{g}_{[k]}^{x} & =\mathbf{P}_{[k-1]}^{x} \cdot \mathbf{X}_{[k]}^{x} \cdot\left[\lambda+\mathbf{X}_{[k]}^{x} \cdot \mathbf{P}_{[k-1]}^{x} \cdot \mathbf{X}_{[k]}^{x}\right]^{-1} \\
\mathbf{P}_{[k]}^{x} & =\lambda^{-1} \cdot \mathbf{P}_{[k-1]}^{x}-\mathbf{g}_{[k]}^{x} \cdot \mathbf{X}_{[k]}^{x} \lambda^{-1} \cdot \mathbf{P}_{[k-1]}^{x} \\
\mathbf{W}_{[k]}^{x} & =\mathbf{W}_{[k-1]}^{x}+\left(\alpha_{[k]}^{x} \cdot \mathbf{g}_{[k]}^{x}\right)^{T}
\end{aligned}
$$

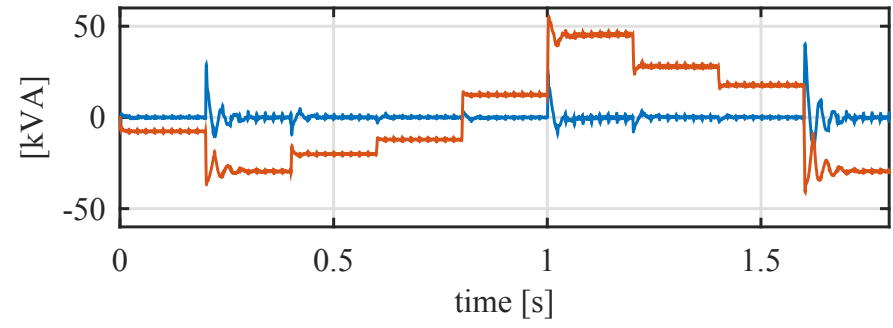

Fig. 8. Simulation results. Active (blue) and reactive (red) power in the VSC2 operated in STATCOM mode.

$$
\begin{gathered}
\mathbf{X}_{[k]}^{x}=\left[i_{\left.g_{[k-1]}\right]}^{x}, i_{g_{[k]}}^{y}, i_{g_{[k-1]}}^{y}, v_{g_{[k]}}^{x}, v_{g_{[k-1]}}^{x}\right]^{T} \\
\mathbf{W}_{[k]}^{x}=\left[a_{1[k]}^{x}, a_{2[k]}^{x}, a_{3[k]}^{x}, b_{0[k]}^{x}, b_{0[k]}^{x}\right]
\end{gathered}
$$

where $\mathbf{P}_{(5 \times 5)}$ is the covariance matrix and it is initialized to $\mathbf{P}=0.01 \cdot \mathbf{I}_{(5 \times 5)} ; \mathbf{g}_{(5 \times 1)}$ is the adaptation gain, and $\lambda=[0,1]$ is the forgetting factor, which need to be selected as a tradeoff of the expected estimation bandwidth and the signal to noise ratio. Values between 0.95 and 1 are often selected. For this paper, the values shown in Table I have been used. After the injection of a new pulse, the estimation of the $\mathbf{W}$ components for both the $\alpha$ and the $\beta$ components is updated and a new estimation for $\mathbf{R}_{\alpha \beta}$ and $\mathbf{L}_{\alpha \beta}$ is obtained using (12).

The algorithm has been considered to be fast enough to be implemented on medium performance digital signal controllers as has been demonstrated in [29].

The simulation results for the impedance estimation using the RLS algorithm under the setup in Fig. 1 are shown in Fig. 8 and 9. As shown, the individual components react to the STATCOM commands as expected.

\section{EXPERIMENTAL RESULTS}

In order to prove the performance of the technique, three different experiments were carried out in the experimental setup of Fig. 10. The converter was operated with the system shown in Fig. 10 under different configurations: 1) Converter VSC 1 feeding a load, isolated from the grid. In this configuration, the DC-side was kept constant at its nominal value of $750 \mathrm{~V}$ by an independent source. 2) Converter VSC 2 connected to grid in STATCOM mode. DC-link is controlled by the d-axis component whereas the pulses are injected at the q-axis. The estimation for different impedances was also performed. 3) Converter connected as the schematic presented in Fig. 1 with converter VSC 2 injecting reactive current steps in the grid, in order to evaluate the effect over the estimation technique. Fig. 11 shows the voltage and current waveforms of VSC1 with the pulsed injection signal when both converters are connected in parallel (configuration 3), and the current resulted from that injection. For all the three cases the same load connected to the PCC is used. Results are exposed in Fig. 12. All values used are presented in Table I.

Figure 12 presents the results of the estimator under real conditions. The left column shows the results for the first case, where the VSC 1 was connected only to a $18 \Omega$ load. The 


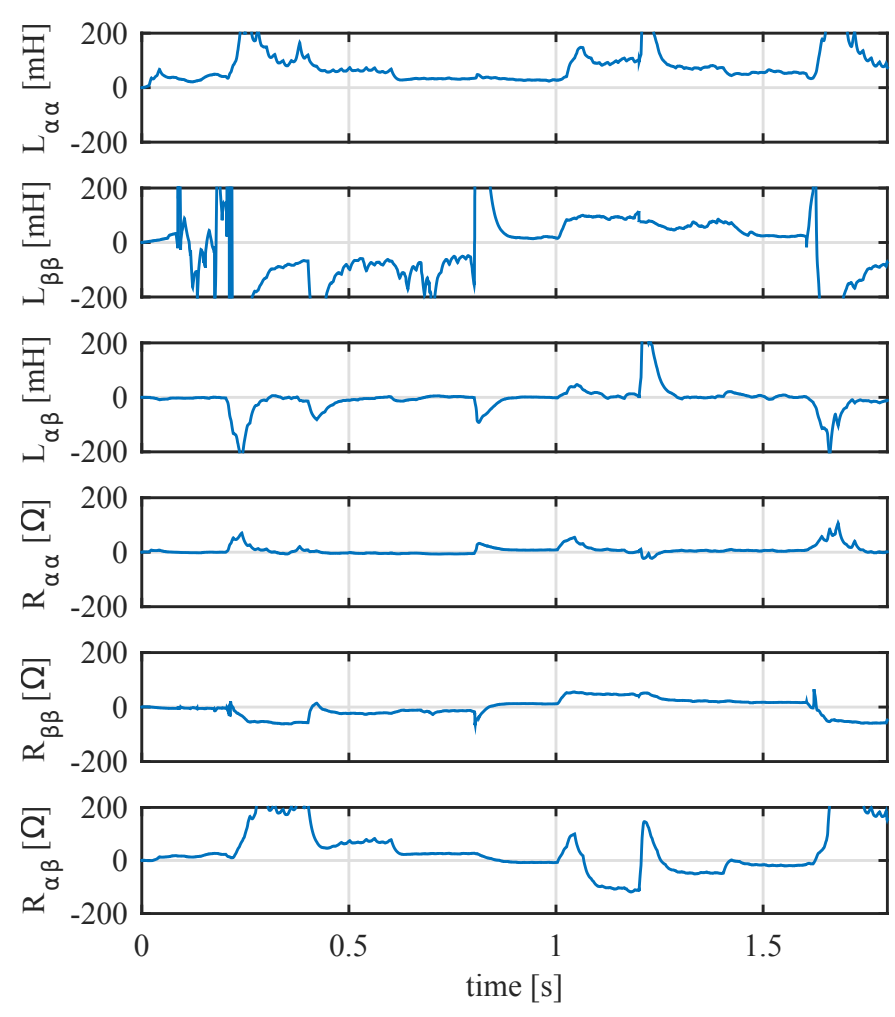

Fig. 9. Simulation results. RLS Identified impedance components.

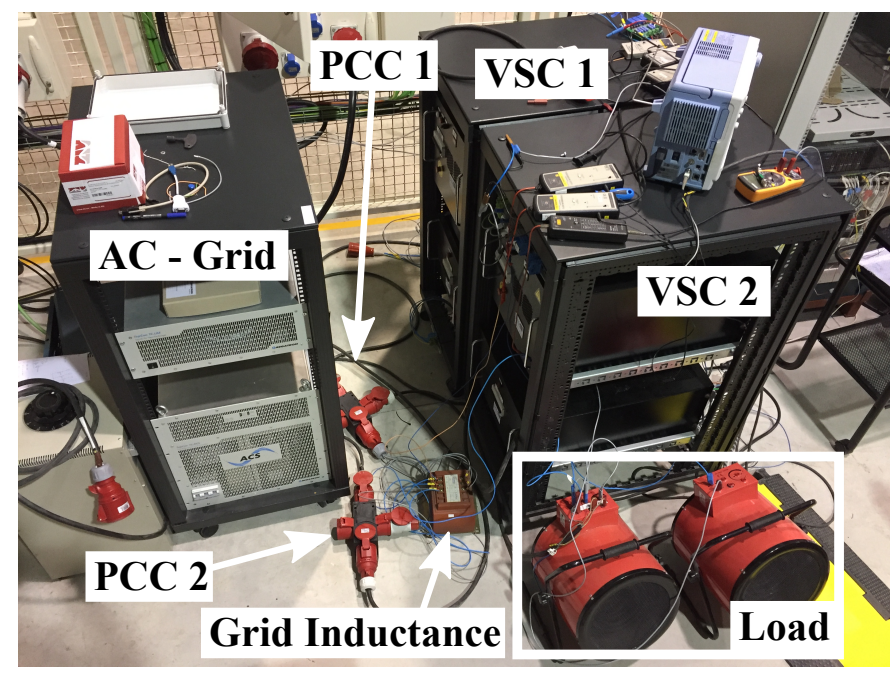

Fig. 10. Experimental setup according to the schematic shown in Fig. 1. At the left side the AC Grid emulator is shown. Converters VSC1 and VSC2 are shown at the right. Two resistive loads are used for varying the estimated impedance.

upper graph shows the $\alpha, \beta$ resistance and the down graph the $\alpha, \beta$ inductance. During this experiment the impedance of the system was kept constant. The second graph shows the performance of the estimation method when its operated as a grid-tied converter while keeping the same load. At $t=1.1 \mathrm{~s}$, the load is changed inducing an unbalance condition in phase A $(18 \Omega \rightarrow 9 \Omega)$. The estimator is able to perceive the change and is reflected in the resistance part, changing the resistance value

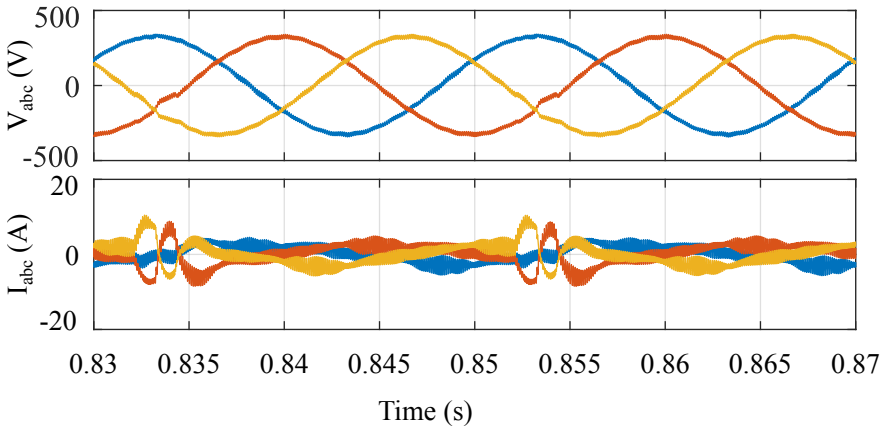

Fig. 11. Voltage and Current Waveforms of converter VSC1 with the pulse injection signal with both converters connected in parallel (configuration 3).

from 18 to $9 \Omega$. In the third case, VSC 1 is connected in parallel with VSC 2, including an impedance between both in order to emulate the grid. VSC2 is injecting $2 A$ reactive current steps each two seconds. Current injection starts at 0.2 and at 0.9 seconds the same unbalance described for previous experiment is applied. For this case, it is clear that the inductive component of the impedance estimation is reacting to the changes.
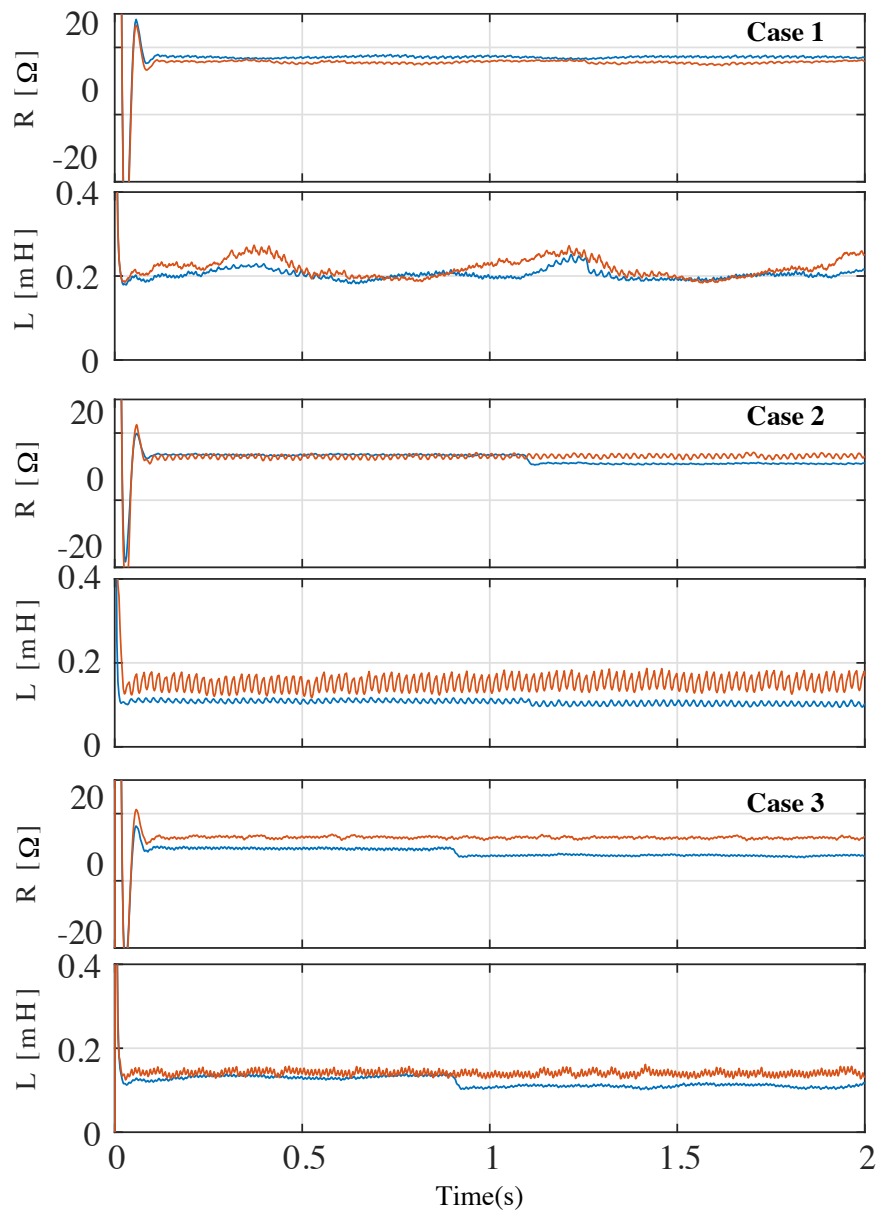

Fig. 12. Experimental Results obtained from the estimation of the $\alpha \beta$ components of the impedance. From top to bottom, 1) VSC 1 feeding an isolated load; 2) VSC1 connected to a AC Grid emulator; 3) VSC1 and VSC 2 connected in parallel 
TABLE I

PARAMETERS

\begin{tabular}{c|c}
\hline Nominal parameters & Value \\
\hline$R_{1} ; R_{2}[\Omega]$ & 0.33 \\
$R_{\text {Load }}[\Omega]$ & 18 \\
$L_{1} ; L_{2}[\mathrm{mH}]$ & 1.7 \\
$L_{\text {Grid }}[\mathrm{mH}]$ & 0.879 \\
pulse mag. [p.u] & 0.1 \\
pulse width. [ms] & 2 \\
$\lambda$ & $0.9 / 0.8$ \\
\hline
\end{tabular}

\section{Conclusions}

This paper has proposed an online grid impedance estimation technique based on a RLS estimator, able to perform an accurate estimation under unbalanced conditions and with other power converter operating in STATCOM mode connected to the same grid. An analytical model for determine the grid impedance both with passive and active loads has been built. The model was used within the RLS algorithm for the parameters estimation. The validity of the proposal has been demonstrated with both simulation and preliminary experimental results.

\section{REFERENCES}

[1] H. Xiao, X. Qu, S. Xie, and J. Xu, "Synthesis of active damping for grid-connected inverters with an LCL filter," in 2012 IEEE Energy Conversion Congress and Exposition (ECCE), Sep. 2012, pp. 550-556.

[2] J. Xu, S. Xie, and T. Tang, "Active Damping-Based Control for GridConnected -Filtered Inverter With Injected Grid Current Feedback Only," IEEE Transactions on Industrial Electronics, vol. 61, no. 9, pp. 47464758, Sep. 2014.

[3] Z. Staroszczyk, "A method for real-time, wide-band identification of the source impedance in power systems," IEEE Transactions on Instrumentation and Measurement, vol. 54, no. 1, pp. 377-385, Feb 2005.

[4] M. Sumner, B. Palethorpe, D. W. P. Thomas, P. Zanchetta, and M. C. D. Piazza, "A technique for power supply harmonic impedance estimation using a controlled voltage disturbance," IEEE Transactions on Power Electronics, vol. 17, no. 2, pp. 207-215, Mar 2002.

[5] M. Liserre, F. Blaabjerg, and R. Teodorescu, "Grid Impedance Estimation via Excitation of LCL -Filter Resonance," IEEE Transactions on Industry Applications, vol. 43, no. 5, pp. 1401-1407, 2007.

[6] A. Vidal, A. G. Yepes, F. D. Freijedo, O. López, J. Malvar, F. Baneira, and J. Doval-Gandoy, "A method for identification of the equivalent inductance and resistance in the plant model of current-controlled gridtied converters," IEEE Transactions on Power Electronics, vol. 30, no. 12, pp. 7245-7261, Dec 2015.

[7] A. K. Broen, M. Amin, E. Skjong, and M. Molinas, "Instantaneous frequency tracking of harmonic distortions for grid impedance identification based on kalman filtering," in 2016 IEEE 17th Workshop on Control and Modeling for Power Electronics (COMPEL), June 2016, pp. 1-7.

[8] A. Bechouche, D. O. Abdeslam, H. Seddiki, and A. Rahoui, "Estimation of equivalent inductance and resistance for adaptive control of threephase pwm rectifiers," in IECON 2016 - 42nd Annual Conference of the IEEE Industrial Electronics Society, Oct 2016, pp. 1336-1341.

[9] A. Ghanem, M. Rashed, M. Sumner, M. A. Elsayes, and I. I. I. Mansy, "Grid impedance estimation for islanding detection and adaptive control of converters," IET Power Electronics, vol. 10, no. 11, pp. 1279-1288, 2017.

[10] D. Martin and E. Santi, "Auto tuning of digital deadbeat current controller for grid tied inverters using wide bandwidth impedance identification," in 2012 Twenty-Seventh Annual IEEE Applied Power Electronics Conference and Exposition (APEC), 2012, pp. 277-284.

[11] L. Asiminoaei, R. Teodorescu, F. Blaabjerg, and U. Borup, "A digital controlled PV-inverter with grid impedance estimation for ENS detection," IEEE Transactions on Power Electronics, vol. 20, no. 6, pp. 14801490, 2005.
[12] D. Reigosa, F. Briz, C. Charro, P. Garcia, and J. Guerrero, "Active Islanding Detection Using High-Frequency Signal Injection," IEEE Transactions on Industry Applications, vol. 48, no. 5, pp. 1588-1597, 2012.

[13] D. Reigosa, F. Briz, C. Blanco, P. Garcia, and J. Manuel Guerrero, "Active Islanding Detection for Multiple Parallel-Connected InverterBased Distributed Generators Using High-Frequency Signal Injection," IEEE Transactions on Power Electronics, vol. 29, no. 3, pp. 1192-1199, 2014.

[14] L. Asiminoaei, R. Teodorescu, F. Blaabjerg, and U. Borup, "A digital controlled pv-inverter with grid impedance estimation for ens detection," IEEE Transactions on Power Electronics, vol. 20, no. 6, pp. 1480-1490, Nov 2005.

[15] T. Roinila, M. Vilkko, and J. Sun, "Online grid impedance measurement using discrete-interval binary sequence injection," in 2013 IEEE 14th Workshop on Control and Modeling for Power Electronics (COMPEL), June 2013, pp. 1-8.

[16] M. Cespedes and J. Sun, "Three-phase impedance measurement for system stability analysis," in 2013 IEEE 14th Workshop on Control and Modeling for Power Electronics (COMPEL), June 2013, pp. 1-6.

[17] M. A. Azzouz and E. F. El-Saadany, "Multivariable grid admittance identification for impedance stabilization of active distribution networks," IEEE Transactions on Smart Grid, vol. 8, no. 3, pp. 1116-1128, May 2017.

[18] M. Cespedes and J. Sun, "Adaptive control of grid-connected inverters based on online grid impedance measurements," IEEE Transactions on Sustainable Energy, vol. 5, no. 2, pp. 516-523, April 2014.

[19] M. Céspedes and J. Sun, "Online grid impedance identification for adaptive control of grid-connected inverters," in 2012 IEEE Energy Conversion Congress and Exposition (ECCE), Sept 2012, pp. 914-921.

[20] M. Sumner, B. Palethorpe, and D. Thomas, "Impedance measurement for improved power quality-Part 1: the measurement technique," IEEE Transactions on Power Delivery, vol. 19, no. 3, pp. 1442-1448, 2004.

[21] N. Hoffmann and F. Fuchs, "Minimal Invasive Equivalent Grid Impedance Estimation in Inductive \#x2013; Resistive Power Networks Using Extended Kalman Filter," IEEE Transactions on Power Electronics, vol. 29, no. 2, pp. 631-641, 2014.

[22] X. Guo, Z. Lu, X. Sun, H. Gu, and W. Wu, "New grid impedance estimation technique for grid-connected power converters," Journal of Engineering Research, vol. 2, no. 3, p. 19, 2014. [Online]. Available: http://dx.doi.org/10.7603/s40632-014-0019-7

[23] L. Asiminoaei, R. Teodorescu, F. Blaabjerg, and U. Borup, "Implementation and Test of an Online Embedded Grid Impedance Estimation Technique for PV Inverters," IEEE Transactions on Industrial Electronics, vol. 52, no. 4, pp. 1136-1144, 2005.

[24] A. Timbus, P. Rodriguez, R. Teodorescu, and M. Ciobotaru, "Line Impedance Estimation Using Active and Reactive Power Variations," in IEEE Power Electronics Specialists Conference, 2007. PESC 2007, 2007, pp. 1273-1279.

[25] A. Riccobono, S. K. A. Naqvi, A. Monti, T. Caldognetto, J. Siegers, and E. Santi, "Online wideband identification of single-phase ac power grid impedances using an existing grid-tied power electronic inverter," in 2015 IEEE 6th International Symposium on Power Electronics for Distributed Generation Systems (PEDG), June 2015, pp. 1-8.

[26] T. Roinila, T. Messo, and A. Aapro, "Impedance measurement of three phase systems in dq-domain: Applying mimo-identification techniques," in 2016 IEEE Energy Conversion Congress and Exposition (ECCE), Sept 2016, pp. 1-6.

[27] A. Suárez-González, P. García, Á. Navarro-Rodríguez, G. Villa, and J. M. Cano, "Sensorless unbalance correction as an ancillary service for lv 4-wire/3-phase power converters," in 2017 IEEE Energy Conversion Congress and Exposition (ECCE), Oct 2017, pp. 4799-4805.

[28] A. Arriagada, J. Espinoza, J. Rodriguez, and L. Moran, "On-line filtering reactance identification in voltage-source three-phase active-front-end rectifiers," in The 29th Annual Conference of the IEEE Industrial Electronics Society, 2003. IECON '03, vol. 1, Nov. 2003, pp. 192-197 vol.1.

[29] P. García, J. M. Guerrero, J. García, A. Navarro-Rodríguez, and M. Sumner, "Low frequency signal injection for grid impedance estimation in three phase systems," in 2014 IEEE Energy Conversion Congress and Exposition (ECCE), Sept 2014, pp. 1542-1549. 